\title{
Development of an Inversion Method for Low Velocity Medium
}

\author{
Afnimar, Andri Dian Nugraha \& Ahmad Syahputra \\ Faculty of Mining and Petroleum Engineering, Bandung Institute of Technology, \\ Jalan Ganesa 10, Bandung 40132, Indonesia \\ Email: afnimar.1987@gmail.com
}

\begin{abstract}
The main problem with the inversion of a low velocity medium is the application of an appropriate ray tracing method after choosing a suitable model parameterization. Block parameterization is not suitable, because it is not capable of representing the velocity model well. A large amount of blocks with a small grid size are needed to express the model well, but in that case, a ray coverage problem will be encountered. A knot-point parameterization model is better suited than a block model, because it can express the velocity model well, while the number of variables is much smaller. Ray calculation using the pseudobending method is not appropriate for the velocity model because of an instability problem at high velocity gradients. The crucial problem of this method involves the initial ray-path that is optimized in order to obtain the "true" ray, but does not satisfy the Fermat principle. These problems can be solved by applying the eikonal-solver method, because this can handle high-velocity gradients and does not need an initial ray path. Using a suitable model parameterization and appropriate ray tracing method, the inversion can obtain good results that fit the desired output. Applying a block model and the pseudobending method will not produce the desired output.
\end{abstract}

Keywords: Eikonal-solver method; Fermat principle; knot-point parameterization; low velocity structure; pseudo-bending method.

\section{$1 \quad$ Introduction}

Many current inversion techniques use seismic wave arrival time data to estimate subsurface structures [1-3]. This is preceded by an effective model parameterization and followed by the selection of an appropriate method of theoretical ray calculation acting as a forward modeling. After that, a system of equations in the form of a matrix can be formed and its inversion can be calculated by various methods.

When model Parameterization is performed to describe a subsurface structure, the chosen number of parameters should not be too high, so as to get a kernel matrix that is not too large and doesn't contain too many zero-value matrix components. Until now, parameterizations usually are carried using a block model, but a large number of blocks are needed to describe the geological

Received November $28^{\text {th }}, 2012$, Revised February $14^{\text {th }}, 2013$, Accepted for publication March $20^{\text {th }}, 2013$.

Copyright (C) 2013 Published by ITB Journal Publisher, ISSN: 2337-5760, DOI: 10.5614/j.math.fund.sci.2013.45.1.8 
structure well. Therefore, an alternative method to parameterize the model is needed. In this study, model parameterization will be conducted using a knot point model as well as a block model. So far, the knot point model has been applied by few seismologists [4]. Both methods of parameterization will be applied to the same subsurface structure, selected according to the criteria mentioned above. Miyazawa and Kato [5] noted that using the velocity and slowness at knot points will produce different tomography result, unless a carefully formulated tomographic problem is applied. We will apply slowness parameterization because in the way we will get the linear form of the kernel function.

The next step is the selection of a ray tracing method suitable for the selected model parameterization. Application of the pseudo-bending method ([6]) is very popular because of its efficiency. However, this method has the disadvantage that it needs an initial value (initial trajectory). This will lead to inaccuracies in the calculation of the "real" trajectory as well as its traveltime, especially in low velocity zones. In addition, there is an instability problem in the ray calculation in case of high-velocity gradient structures. In this study, we have analyzed the problems of the pseudo-bending method and have found another ray tracing method, which does generate an accurate calculation. This method is called the eikonal-solver method ([7]), where an initial value (initial trajectory) is not required and the problems with high velocity gradients can be overcome. The selection criterion for choosing the appropriate method is that is has to satisfy the Fermat principle, according to which the rays should avoid low-velocity zones.

The last step is the formulation of the inversion formula using a gradient inversion method. The inversion method that we have developed can be applied to a highly heterogeneous medium, especially in cases where volcanic seismic low-velocity anomalies exist in weak zones and zones of high fluid content. We have compared the method with the inversion method that is most commonly employed and that uses a block model and pseudo bending method. In this article, the superiority of our method will be demonstrated.

This method has not yet been applied to real data because of the unavailability of $2 \mathrm{D}$ field data. In the near future, the method will be further developed for 3D cases and applied to data of some volcanoes in Indonesia. 


\section{The Method}

\subsection{Model Parameterization}

Model parameterization or definition of estimated variables, preceding the inversion process is an essential first step, because if this is not done appropriately, the results will not properly describe the subsurface. In other words, the inversion result must satisfy the physical and geological principles. In the case of parameterization for a subsurface structure, previous geological and geophysical studies provide important preliminary data. From these data, we do not necessarily need to create a block model; a 1D model or a boundary layer model may be is enough. However, in the case of something like a volcanic structure, a block model or knot-point model is more suitable, because of its heterogeneity, which makes the layers of the structure difficult to define. In the block model, the constant velocity or slowness is defined for each block, while in the knot-point model, the velocity or slowness is defined at knot point. In this study, slowness at a point $s(x, z)$ in the knot-point model is defined as a linear interpolation ([2]) from four knot points around that point, which can be formulated as

$$
s(x, z)=\sum_{n=i}^{i+1} \sum_{m=k}^{k+1} S_{n, m}\left|\frac{x-x_{n}}{\Delta x} \| \frac{z-z_{m}}{\Delta z}\right|,
$$

where $S_{i, k}$ is the slowness at point $\left(x_{i}, z_{k}\right)$.

\subsection{Pseudo-Bending Method}

This method was developed by Um and Thurber [6], based on the ray path that is represented by a linear interpolation from a number of points. This method requires an initial trajectory. The initial value is optimized to obtain the final trajectory that satisfies the ray equation. This can be done by determining the normal direction of the initial ray trajectories followed by the application of the Fermat principle.

Um and Thurber proposed a three-point scheme that is successively renewed satisfying the Fermat principle along the ray. Figure 1 shows three points $\vec{r}_{i-1}, \vec{r}_{i}$ and $\vec{r}_{i+1}$, as well as the initial-guess point $\vec{r}_{i}^{\text {old }}$. The pseudo-bending method will update the initial-guess point to estimate $\vec{r}_{i}^{\text {new }}$. This estimation is obtained by considering the perturbation $\vec{r}_{\text {mid }}$ which is the midpoint between $\vec{r}_{i-1}$ and $\vec{r}_{i+1}$. The vector $\vec{n}$ that expresses the bending direction is calculated by the formula 


$$
\vec{n}=\frac{\nabla v-\left(\nabla v \cdot \vec{m}_{t}\right) \vec{m}_{t}}{\left|\nabla v-\left(\nabla v \cdot \vec{m}_{t}\right) \vec{m}_{t}\right|}
$$

where

$$
\vec{m}_{t}=\frac{\vec{r}_{i+1}-\vec{r}_{i-1}}{\left|\vec{r}_{i+1}-\vec{r}_{i-1}\right|} .
$$

The distance $R$ along $\vec{n}$ is obtained by minimization or application of the Fermat principle on the traveltime equation.

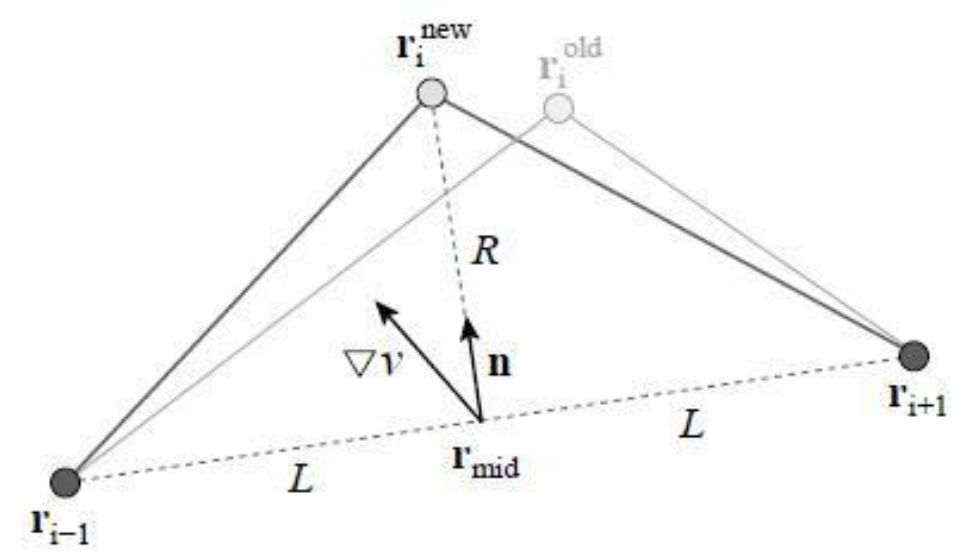

Figure 1 Illustration of the three-point perturbation scheme in the pseudobending method.

\subsection{Eikonal-Solver Method}

Vidale [8,9] proposed a finite difference approximation to the $2 \mathrm{D}$ and $3 \mathrm{D}$ eikonal equations. Of course, this finite difference scheme is applied to the gridded subsurface structure model. Vidale formulation is not accurate for the case of a heterogeneous structure because it can cause multipathing effects from other waves such as a head wave. Afnimar \& Koketsu [7] proposed a scheme to solve this problem by applying the Podvin \& Lecomte operator [10], and also provided illumination for the $2 \mathrm{D}$ case and propose a new operator for the $3 \mathrm{D}$ case with an illumination condition as well.

The result of the implementation of the above scheme is a travel time at each grid point. If it is made its contours, it will get the wavefront. The ray can be calculated by tracing it from the station to the source point, considering that the ray direction or the wave propagation direction is always perpendicular to the wavefront. 


\subsection{Gradient Inversion Method}

The function of seismic wave traveltime $t$ with respect to the defined parameter, in this case the slowness $S_{i, k}$ as contained in Eq. (1), is a nonlinear problem because of the heterogeneity of the earth structure. In the gradient inversion method, a nonlinearity problem can be solved by iteration of the linearized form of the traveltime function [1], which starts from an initial model. The linearization form is

$$
\sum_{k=1}^{K} \sum_{i=1}^{I} \frac{\partial t}{\partial S_{i, k}} \Delta S_{i, k} \sim \Delta t
$$

The traveltime derivative with respect to the slowness at a knot point can be formulated as a chain derivative, which involve Eq. (1)

$$
\begin{aligned}
\frac{\partial t}{\partial S_{i, k}} & =\frac{\partial t}{\partial s(x, z)} \frac{\partial s(x, z)}{\partial S_{i, k}} \\
& =l_{x, z}\left|\frac{x-x_{i}}{\Delta x}\right|\left|\frac{z-z_{i}}{\Delta z}\right|,
\end{aligned}
$$

where $l_{x, z}$ is a ray length at $(x, z)$. In order to ensure that the solution of matrix Eq. (4) is stable, it is necessary to apply damping

$$
\mu \Delta S_{i, k} \sim 0 \text {. }
$$

The combination of Eqs. (4) and (6) can be written in a more compact form

$$
\mathbf{A} \Delta \mathbf{S}=\mathbf{d} .
$$

which can be solved by applying the LSQR method [11]. The uncertainty of the inversion result is calculated from the square root of the diagonal components of the covariance matrix

$$
\mathbf{C}=\frac{\sum_{i}^{N} d_{i}^{2}}{N-M}\left(\mathbf{A}^{T} \mathrm{~A}\right),
$$

where $N$ is the number of data and $M$ is number of parameters [12].

\section{Application to Volcano Structure Model}

In this study, we have tried to explore the application of the method described in section 2 to, the volcano structure as shown in Figure 2 (left). The shape of this 
structure occurs uniquely in the volcano, where there are low-velocity anomalies associated with magma or fluids or weak zones.

\subsection{Model Parameterization}

To obtain an accurate inversion result, we must first define a suitable model parameterization. If the model parameterization is made by using a block model, as is mostly the case in tomography, a large number of blocks is needed to describe the geological model well. If the block size is $0.5 \mathrm{~km}$, as shown in Figure 2 (middle), then the number of variables is 400 . This parameterization is good enough to describe the model, but because of the large number of variables many blocks will not be crossed by rays, which will result many zero values in the kernel matrix. In other words, ray coverage will not be good. If the block size is enlarged, the number of variables will be reduced but the description of the model will be worse. To solve this problem, we propose to apply knot-point model parameterization, as shown in Figure 2 (right). With at distance between knot-points of $2 \mathrm{~km}$, the number of variables is only 36 , whereas the model is described much better, as shown Figure 2 (left). Hence, this parameterization is very effective compared to the block model. Slowness distribution at a certain point is calculated using Eq. (1).
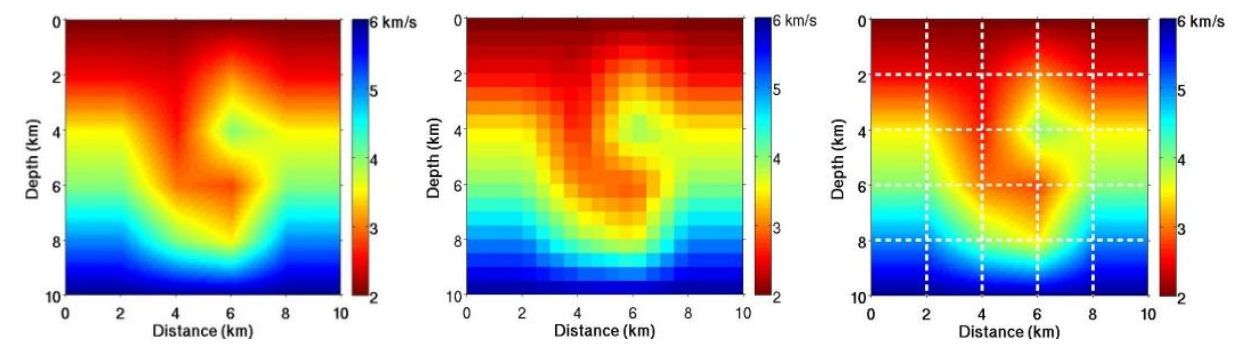

Figure 2 Left: Low velocity model (red). Middle: Parameterizing the model using blocks with a size of $0.5 \mathrm{~km}$. Right: Parameterizing the model using knot points with distance between points of $2 \mathrm{~km}$.

\subsection{Selection of Ray Tracing Method}

Parameter determination as described above is followed by the selection of a suitable ray tracing method. Application of the pseudo-bending method on this model will encounter an instability problem, which occurs in the ray calculation marked by the dashed white line in Figure 3 (left). This is caused by the large velocity gradient. Gradient calculation using Eq. (2) is needed for the minimization of the ray trajectory, but because the gradient is large, it will result in no solution. A serious problem is the inaccuracy of this method, because it does not follow the Fermat principle. For example, rays from a source number 7, see (Figure 3 left), that should "avoid" low-velocity zones and should pass 
the high-velocity zones, where the traveltime will be shorter. Likewise rays from other source points. Another problem with the method is that it requires an initial trajectory. Usually, the initial trajectory is a straight line, as shown in Figure 1. If the velocity structure is not too heterogeneous, the trajectory optimization performed by this method will get the "real" raypath (the one which has the smallest travel time). However, for a very heterogeneous velocity structure, which contains low-velocity anomalies, this is not a good method, because the selection of the initial trajectory close to the actual trajectory is very difficult. As a result, the trajectory obtained will not turn out as expected.
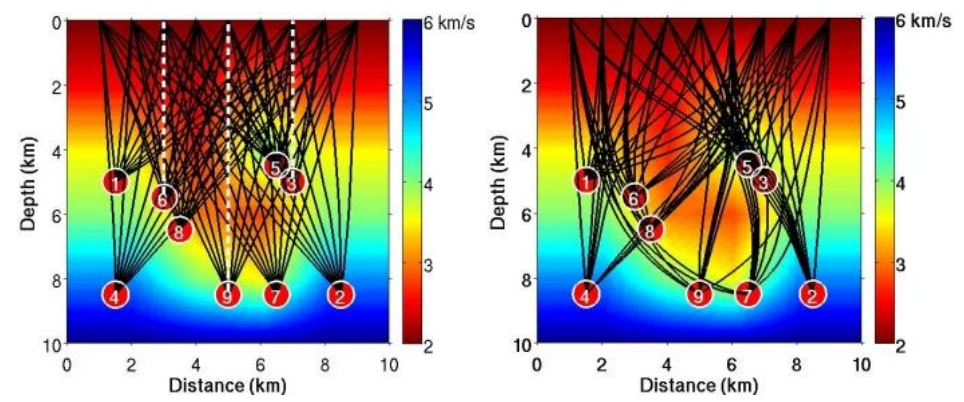

Figure 3 Left: Application of the pseudo-bending method [6]. Right: Application of the eikonal-solver method [7].

To overcome the above problems, in this research, we have applied the eikonalsolver method. The result, as shown in Figure 3 (right), shows that all rays can be calculated properly. The high velocity gradient problem can be solved, because the calculation method does not involve the velocity gradient, as is the case in the pseudo-bending method. Ray trajectories obtained with this method can avoid low-velocity zones and pass high-velocity zones. This satisfies the Fermat principle, according to which the travel path must pass through a zone in the minimum traveltime.

\subsection{Application of Gradient Inversion Method}

As explained in Section 2.4, the concept of this inversion is the minimization of the theoretical traveltime and the observational traveltime, which is done iteratively starting from initial model to get the optimal final model. In order to test the method developed in this study, we have applied to a synthetic data. The observational traveltime is the traveltime calculated in the model in Figure 2 (left). To approach the field conditions, where field data always contain noise, the data were added with Gaussian noise. The initial model applied in the inversion was $1 \mathrm{D}$ model that is commonly used in tomography. It was taken from the left or right side of the velocity model in Figure 2, from $2 \mathrm{~km} / \mathrm{s}$ (on the surface) to $6 \mathrm{~km} / \mathrm{s}$ (at the basement). 
This synthetic model involves nine source points and nine receivers on the surface. Consequently, there are 81 observational data with 36 parameters. Gaussian noise with mean level $\sigma=0.0-0.2$ is included in the observational data. To avoid instability in the matrix inversion, norm damping $\mu=5.0$ was applied. The inversion was performed in eight iterations and the solution was the result whose RMS traveltime difference between two iterations was small enough.

The inversion result using the observational data without noise is shown in Figure 4. The selected result is the fourth iteration, where the RMS value was relatively close to the subsequent iteration result. This result could very well be close to the desired model. The uncertainty is smaller than $0.3 \mathrm{~km} / \mathrm{s}$. The biggest uncertainties are at the bottom of the model, because of little ray coverage compared to the top of the model. For the data with a noise level of 0.1 , the inversion result, see (Figure 5 middle), still shows the desired output. The uncertainty, see (Figure 5 right) is still very good for the low-velocity zone, which is about $0.25 \mathrm{~km} / \mathrm{s}$. For data with higher noise levels, as in Figure 6, the inversion result is less comprehensive compared to the desired output.
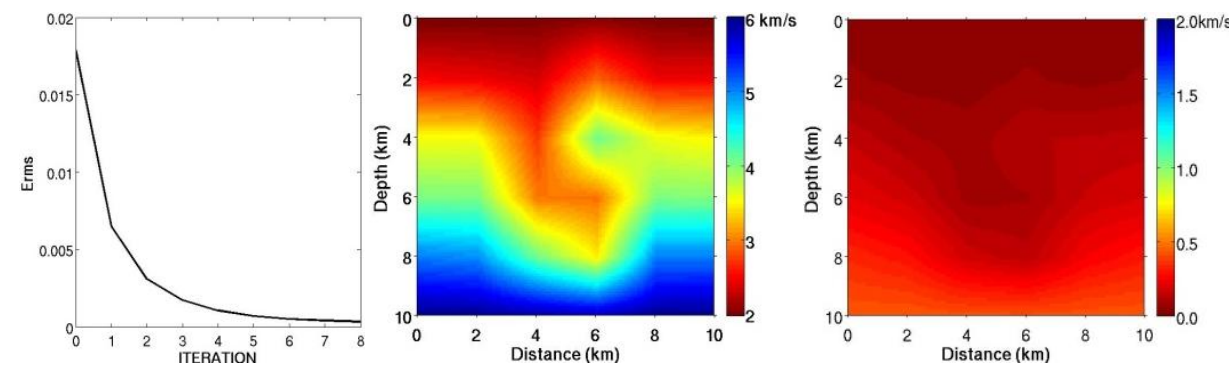

Figure 4 Inversion result with damping $\mu=5.0$ and Gaussian noise level $\sigma=0.0$. Left: RMS values of traveltime residual versus iteration. Middle: Inversion result image at the fourth iteration. Right: Uncertainty of inversion results.
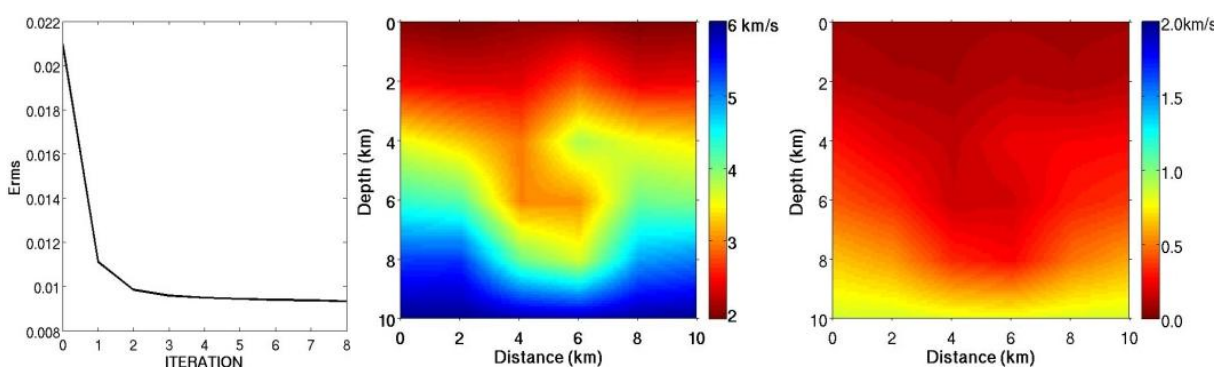

Figure 5 Inversion results with damping $\mu=5.0$ and Gaussian noise level $\sigma=0.1$. Left: RMS travel time residual versus iteration. Middle: Inversion result image at the third iteration. Right: Uncertainty of inversion result. 
Overall, the inversion result can successfully reconstruct the low-velocity zones. The key of this inversion result is the appropriate model parameterization, followed by a suitable method of ray tracing, so the rays can travel through all areas between four knot points.
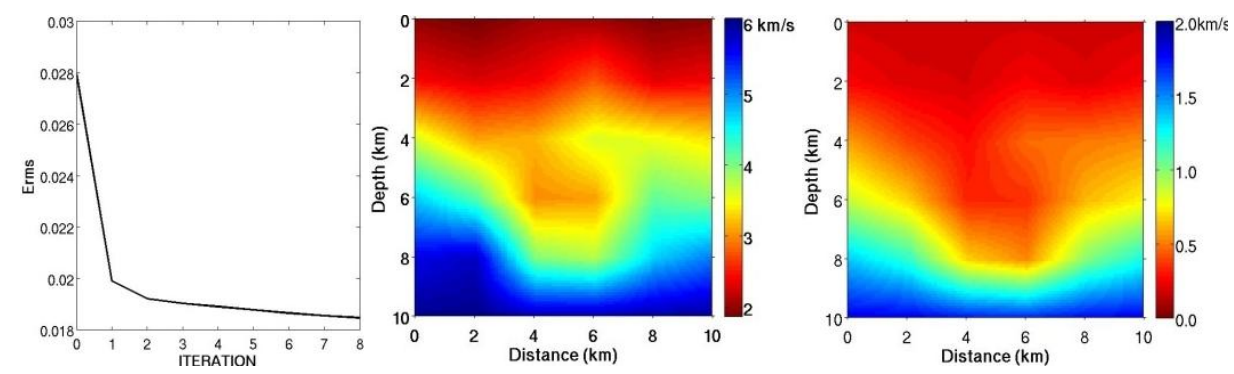

Figure 6 Inversion results with damping $\mu=5.0$ and Gaussian noise level $\sigma=0.2$. Left: RMS value of traveltime residual versus iteration. Middle: Inversion result image at the third iteration. Right: Uncertainty of inversion result.

For comparison, we have also performed an inversion using the same data, but now applying block-model parameterization and calculating the rays using the pseudo-bending method that is commonly applied in tomography. With model parameterization as shown in Figure 2 (middle), the inversion result shown in Figure 7 (left) does not approach the desired output. This is probably caused by the inappropriate ray calculation, as discussed in section 3.2. Attempting to improve the ray coverage, we enlarged the block size to $1 \mathrm{~km}$ and $1.5 \mathrm{~km}$; the inversion results are shown in Figure 7 (middle and right). The low-velocity anomaly in the deep targets could be somewhat resolved, but it doesn't describe the subsurface structure well, because the block size is too large.
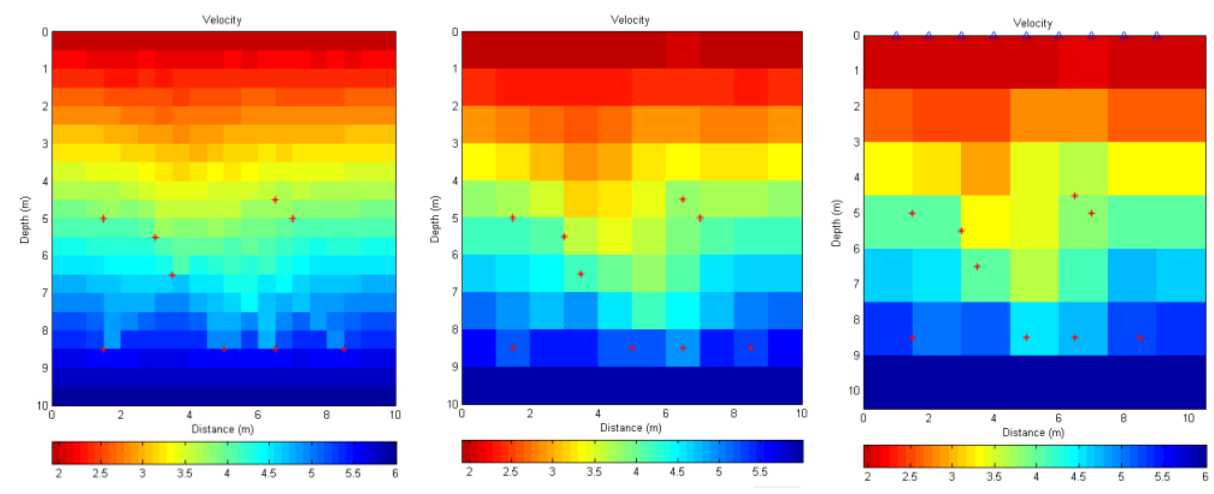

Figure 7 Inversion results with block model and pseudo-bending, damping $\mu=2.0$ and without noise. Left: Inversion result for block size $0.5 \mathrm{~km}$. Middle: Inversion result for block size $1 \mathrm{~km}$. Right: Inversion result for block size 1.5 $\mathrm{km}$. 


\section{Conclusion}

In this study, firstly, an appropriate model parameterization for low velocity zones using knot points has been successfully developed. The knot-point model reduces the number of inversion variables, but it can describe the subsurface structure very well. Secondly, the eikonal-solver method can overcome the problems of the pseudo-bending method with, high-velocity gradients and ray trajectories that do not conform to the Fermat principle. Thirdly, considering these two points, the inversion results of the proposed method can reconstruct low-velocity zones well. This is achieved by appropriately describing the structure and calculating the precise ray tracing. On the other hand, application of the inversion method using a block model and the pseudo-bending method doesn't produce good inversion results.

\section{Acknowledgements}

This research is supported by the Capacity Improvement Research Program ITB 2010. We thank Dr. Shutaro Sekine and the other reviewers for their contribution to improving our manuscript.

\section{References}

[1] Zelt, C.A. \& R.B. Smith, Seismic Traveltime Inversion for 2-D Custal Velocity Structure, Geophys. J. Int, 108, pp. 16-34, 1992.

[2] Afnimar, Koketsu, K. \& Nakagawa, K., Joint Inversion of Refraction and Gravity Data for Three-Dimensional Topography of A Sediment Basement-Interface, Geophys. J. Int., 151, pp. 243-254, 2002.

[3] Nugraha, A.D. \& Mori, J., Three-Dimensional Velocity Structure in The Bungo Channel and Shikoku Area, Japan, and Its Relationship to LowFrequency Earthquakes, Geophys. Res. Lett, 33, L24307. doi:10.1029/ 2006GL028479, 2006.

[4] Zhao, D., Hasegawa, A. \& Horiuchi, S., Tomographic Imaging of Pand S Wave Velocity Structure Beneath Northeastern Japan, J. Geophys. Res., 97, pp. 19909-19928, 1992.

[5] Miyazawa, M. \& Kato, M., On Interpolation Functions in Traveltime Tomography, Geophys. J. Int., 158, pp.169-178, 2004.

[6] Um, J. \& Thurber, C.H., A Fast Algorithm for Two-Point Seismic Ray Tracing, Bull. Seism. Soc. Am., 77, pp. 972-986, 1987.

[7] Afnimar \& Koketsu, K., Finite-Difference Traveltime Calculation for Head Waves Traveling Along an Irregular Interface, Geophys. J. Int, 143, pp. 729-734, 2000.

[8] Vidale, J., Finite-Difference Calculation of Traveltimes, Bull. Seism. Soc. Am., 78, pp. 2062-2076, 1988. 
[9] Vidale, J., Finite-Difference Calculation of Traveltimes in Three Dimensions, Geophysics, 55, pp. 521-526, 1990.

[10] Podvin, P. \& Lecomte, I., Finite-Difference Computation of Traveltimes in Very Contrasted Velocity Models: A Massively Parallel Approach and Its Associated Tools, Geophys. J. Int, 105, pp. 271-284, 1991.

[11] Paige, C.C. \& Saunders, M.A., LSQR: An Algorithm for Sparse Linear Equations and Sparse Least Squares, ACM Trans. Math. Soft., pp. 43-71, 195-209, 1982.

[12] Grandis, H., Pengantar Pemodelan Inversi Geofisika, Himpunan Ahli Geofisika Indonesia (HAGI), 2009. 